\title{
Simultaneous Multiscale Polyaffine Registration by Incorporating Deformation Statistics
}

\author{
Christof Seiler ${ }^{1,2}$, Xavier Pennec ${ }^{2}$, and Mauricio Reyes ${ }^{1}$ \\ 1 Institute for Surgical Technology and Biomechanics, \\ University of Bern, Switzerland \\ 2 Asclepios Research Group, INRIA Sophia Antipolis, France
}

\begin{abstract}
Locally affine (polyaffine) image registration methods capture intersubject non-linear deformations with a low number of parameters, while providing an intuitive interpretation for clinicians. Considering the mandible bone, anatomical shape differences can be found at different scales, e.g. left or right side, teeth, etc. Classically, sequential coarse to fine registration are used to handle multiscale deformations, instead we propose a simultaneous optimization of all scales. To avoid local minima we incorporate a prior on the polyaffine transformations. This kind of groupwise registration approach is natural in a polyaffine context, if we assume one configuration of regions that describes an entire group of images, with varying transformations for each region. In this paper, we reformulate polyaffine deformations in a generative statistical model, which enables us to incorporate deformation statistics as a prior in a Bayesian setting. We find optimal transformations by optimizing the maximum a posteriori probability. We assume that the polyaffine transformations follow a normal distribution with mean and concentration matrix. Parameters of the prior are estimated from an initial coarse to fine registration. Knowing the region structure, we develop a blockwise pseudoinverse to obtain the concentration matrix. To our knowledge, we are the first to introduce simultaneous multiscale optimization through groupwise polyaffine registration. We show results on 42 mandible CT images.
\end{abstract}

\section{Introduction}

Mandibular fractures most commonly result from facial trauma, with close to half of the patients requiring surgical repair [6]. A majority of $75 \%$ of fractures occur in males aged between 20 and $30 \underline{6}$, and are often caused by physical assault. For these cases surgical repair proofs most effective, with the goal of recovering the anatomical structure prior to the injury and thus restoring normal function. To reach this goal the surgeon places wires or implants at the fracture site, so that the natural fusion of separated bone pieces restores the prior structure as closely as possible. A correct repair aligns teeth for food intake, and restores the patient's aesthetics. In [12], the authors propose a classification scheme for mandibles based on regions according to anatomical, functional and aesthetic considerations. The online register www.aofoundation.org uses the 

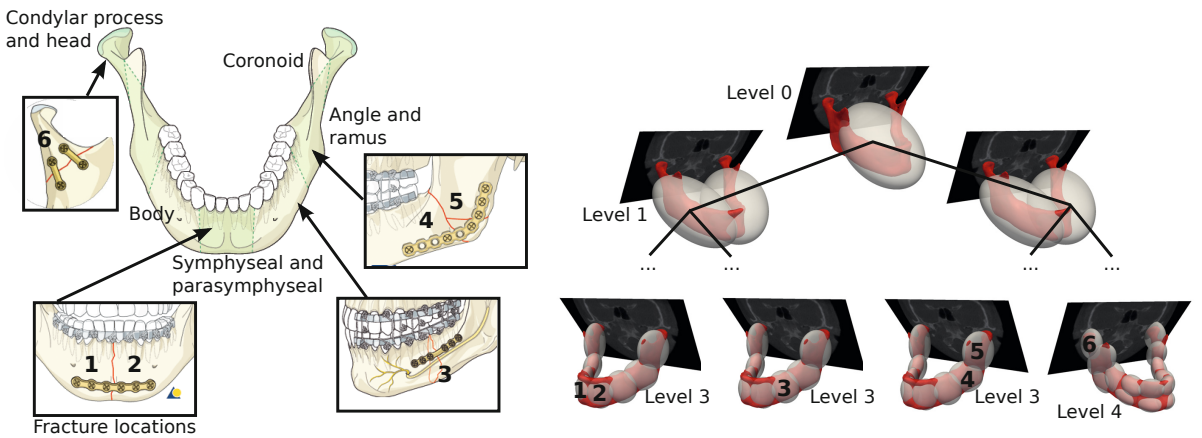

Fig. 1. Left: Subdivision of mandible into anatomical regions proposed by the AO foundation to classify fractures for reconstructive surgery. Implants at four different anatomical sites are shown. Images source: www.aofoundation.org Right: Tree of Gaussian weights. Each Gaussian weight represents one region and is visualized as one ellipsoids at $\sigma$. The contours extracted from CT data in red and one slice of the original CT image (to indicate that we work in the image domain and not only on the contour) are shown.

same classification (Fig. 1) to guide surgeons through the major steps of mandible reconstructive surgery ranging from diagnosis, selection of the optimal surgical approach, to aftercare treatment. In addition to this classification scheme, the mandible can be subdivided even further into smaller regions, e.g. one region for each tooth. In the image space, this subdivision can theoretically be performed up to the voxel level, where coarser levels enclose finer ones, representing a hierarchy of regions that can be organized in a tree-like fashion. As the regions become more fine, it is harder to find a consensus among clinicians on the size, shape and location of the region.

Recent work on biomechanical analysis of implants indicates that geometry and topology of implants are crucial to fracture stability [7]. Due to the high economical cost of patient specific implants current approaches focus on population-based implant design. Common key steps to population-based design, e.g. [3], are registration to capture shape variability as encountered in a population and statistical analysis of the registration results, performed subsequently and independently.

Due to region-based description of the mandible shape and the need for volumetric information we focus on locally affine transformations, also called polyaffine transformations. Polyaffine transformations fuse locally rigid and affine transformations into a diffeomorphism [1. An efficient registration algorithm using approximations of polyaffine transformations was presented in [5]. To consider more complex shapes and foster reusability, 44 presented a multiscale approach with affine regions defined using a data-driven approach. The method splits rectangular shaped regions, which are aligned along the image directions, only if certain conditions are met. The authors in [11] iteratively optimize between affine parameters and anchor positions (center of regions) estimation, 
through an expectation maximization approach. Even though these methods are very promising, the link between the clinical regions (Fig. 1) and the regions found by these algorithms, is either constrained by aligned rectangular shaped regions [4] or produce an intractable number (around 500) of regions [11. In [10], the authors introduced a hierarchical multiscale tree structure (called polyaffine transformation trees, or PolyTree in short) that is motivated by the nature of the mandible anatomy, where regions are ordered and interact with each other in a way tractable for human understanding. Aforementioned polyaffine methods are pairwise registrations, and to our knowledge, no groupwise registration (e.g. [9] ) has been proposed in this context. We believe that transformations on a groupwise level are crucial. This is motivated by the assumption that there should be one configuration of regions that describes an entire group of images, with varying transformations for each region. Furthermore, the multiscale property of mandibles and other anatomical structures should be incorporated.

In [2], the authors presented a Bayesian approach for affine registration, in this paper, we propose a Bayesian formulation of polyaffine registration across scales. We reformulate PolyTrees in a probabilistic way. We introduce a generative statistical model, which enables us to incorporate deformation statistics as a prior in a Bayesian setting. We find optimal transformations by optimizing the maximum a posteriori probability (MAP). In Section 2, we describe PolyTrees, which were recently introduced in [10, to describe intersubject deformations. In Section 3, we reformulate PolyTrees in a probabilistic way and show how to find optimal transformations using MAP estimates with groupwise deformation statistics as a prior. In Section 4, we show results on 42 mandible CT images.

\section{Multiscale Description of Intersubject Deformations}

In this section we reinterpret polyaffine transformation trees (PolyTree) recently introduced in [10]. PolyTrees are parametric transformation that describe nonrigid deformations with a low number of parameters and captures the shape variability of multiscale structures. First, we introduce the tree structure, second we define data-driven regions, and lastly, we show the estimation of transformations using the log-demons. The difference with [10] is that we reformulate the equations in vectorized form: Vect is the column-wise vectorization of a matrix $m=\operatorname{Vect}(\mathrm{M})$, where $m$ is a vector and $M$ a matrix. The motivation for this will become evident in Section 3 .

Structuring of Locally Affine Transformations in Trees. For $N$ regions let $M=\left[M_{1} \ldots M_{N}\right]^{T}$ be the $3 \times 4 N$ non null components of the matrix logarithm of affine transformations and $v_{M}(x)$ be the parametrized polyaffine stationary velocity field:

$$
v_{M}(x)=M(w(x) \otimes \tilde{x}), \log \left(\left[\begin{array}{cc}
A_{i} & t_{i} \\
0 & 1
\end{array}\right]\right)=\left[\begin{array}{c}
M_{i} \\
0
\end{array}\right],
$$

where $\tilde{x}=\left[\begin{array}{ll}x & 1\end{array}\right]^{T}, x$ is the spatial position, $w(x)=\left[w_{1}(x) \ldots w_{N}(x)\right]^{T}, \otimes$ is the Kronecker product, $A_{i}$ is the affine transformation matrix and $t_{i}$ is the 
translation vector. The weights are normalized, $\forall x \in \Omega: \sum_{i=1}^{n} w_{i}(x)=1$, and structured in a tree-like fashion as depicted in Fig. 1, this enables to describe multiscale deformations.

Data-Driven Definition of Regions. The spatial weight functions $w_{i}(x)$ are defined as multivariate Gaussian distributions, $w_{i}(x)=\mathcal{N}\left(\bar{x}_{i}, \Xi_{i}\right)$. The parameters of the distributions are found by applying the oriented bounding box algorithm (OBBTree) to the feature image, $\psi(x)=\log \left(1+\left\|\nabla I_{t}\right\|^{2}\right)$, where $\nabla I_{t}$ is the gradient of template image. We take the logarithm of the gradient to be more robust against small changes in intensities due to noise. The feature-weighted barycenter of region $\Omega_{i}$ (discretized at voxel indices $j$ ), $\bar{x}_{i}=$ $\left(\sum_{j \in \Omega_{i}} \psi\left(x_{j}\right)\right)^{-1} \sum_{j \in \Omega_{i}^{l}} \psi\left(x_{j}\right) x_{j}$, and the feature-weighted covariance matrix of the region, $\Xi_{i}=\left(\sum_{j \in \Omega_{i}^{l}} \psi\left(x_{j}\right)\right)^{-1} \sum_{j \in \Omega_{i}^{l}} \psi\left(x_{j}\right)\left(x_{j}-\bar{x}_{i}^{l}\right)\left(x_{j}-\bar{x}_{i}^{l}\right)^{T}$. Following the same strategy as in the original OBBTree algorithm, the region is split at the feature-weighted mean point orthogonal to the first principle component and the splitting procedure is recursively repeated in the two new created subregions. This step is identical to the method proposed in [10.

Estimation of Transformations with the Log-Demons Algorithm. The general form of the log-demons algorithm for stationary velocity fields (SVF) was formulated in [13. The goal is to find $v_{M}$ that warps the fixed image $I_{t}$ into the moving image $I_{s}$ (or resamples $I_{s}$ in $I_{t}$ ), by minimizing the cost functional, $C\left(I_{t}, I_{s}, v, v_{M}\right)=\sigma_{i}^{-2} \operatorname{Sim}\left(I_{t}, I_{s}, v_{M}\right)+\sigma_{x}^{-2} \operatorname{dist}\left(v, v_{M}\right)^{2}+\sigma_{T}^{-2} \operatorname{Reg}(v)$, where Sim, dist and Reg, are the similarity, the hidden and the regularization term, respectively. Each term has a weighting parameter $\sigma_{i}, \sigma_{x}$ and $\sigma_{T}$. As shown in 13. $C\left(I_{t}, I_{s}, v, v_{M}\right)$ can be optimized alternatively over the variables $v$ and $v_{M}$. Given $v$, the correspondence velocity field computed by the first optimization part of the log-demons algorithm, we solve for $M$ using linear least squares, i.e. minimizing $C(M)=\int_{\Omega} \lambda(x)\|v(x)-M(w(x) \otimes \tilde{x})\|^{2} d x$, where $\lambda$ is a binary mask indicating background voxels (if no mask is available $\forall x \in \Omega: \lambda(x)=1$ ). In contrast to the original formulation in [10 the transformations parameters $M$ are vectorized, and the Kronecker product is used. This reformulation is necessary for the next section, where we present a generative statistical model, which enables us to incorporate deformation statistics as a prior in a Bayesian setting. We find optimal transformations by optimizing the MAP.

\section{Incorporating Deformation Statistics}

In this section we present the MAP to find optimal transformations with and without prior for $K$ patients. We denote $\hat{m}_{k}$ as the $k$ th transformation found with registration without prior, and $\check{m}_{k}$ with prior. We denote $[k]$ to consider all patients.

Generative Statistical Model of Polyaffine Transformation Trees. Here we make two assumptions: First, we assume that the velocities are independent 
at all voxel positions within the mask $\Omega$. This is an approximation in the case of the log-demons algorithm, where the voxels of correspondence field $v$ are not entirely independent due to the natural smoothness of the images used. Second, we assume the independence of the velocities across subjects. This is only the case if the template with which the patients are registered is unbiased. With these assumption we end up with the log likelihood (modulo unnecessary constants),

$$
\mathcal{L}\left(v^{[k]} \mid m^{[k]}\right)=-\sum_{k} \log \left(P\left(v^{k} \mid m^{k}\right)\right)=\frac{1}{2 \sigma_{v}^{2}} \sum_{k}^{K} \int_{\Omega} \lambda(x)\left\|v^{k}(x)-v_{M^{k}}(x)\right\|^{2} d x,
$$

with the underlying Gaussian noise model $v^{k}(x)=v_{M^{k}}(x)+\mathcal{N}\left(0, \sigma_{v}^{2} I_{3}\right)$, where $I_{3}$ is the $3 \times 3$ identity matrix, and $K$ subjects. For one patient, the probability to observe $v^{k}$ given $m^{k}$ is,

$$
P\left(v^{k} \mid m^{k}\right) \propto \exp \left(-\frac{1}{2}\left(m^{k}-\hat{m}^{k}\right)^{T} \Sigma\left(m^{k}-\hat{m}^{k}\right)\right),
$$

where $\hat{m}^{k}$ denotes the optimal transformation parameters without a prior on $m^{k}$. To find the optimal solution we take the derivative of $\mathcal{L}\left(v^{k} \mid m^{k}\right)$ with respect to $m^{k}$ and set it to zero,

$$
\begin{aligned}
\hat{m}^{k} & =\Sigma^{-1} b^{k}, \\
\Sigma & =\frac{1}{2 \sigma_{v^{2}}} \int_{\Omega} \lambda(x)\left(w(x) w(x)^{T}\right) \otimes\left(\tilde{x} \tilde{x}^{T}\right) d x \otimes I_{3}, \\
b_{k} & =\frac{1}{2 \sigma_{v^{2}}} \int_{\Omega} \lambda(x)(w(x) \otimes \tilde{x}) \otimes v^{k}(x) d x,
\end{aligned}
$$

where $d x$ is a scalar.

Maximum Likelihood Estimation with Prior on the Transformations. Now we show how to find $\check{m}^{k}$ transformations with a prior. Assume that $m^{k} \sim$ $\mathcal{N}(\bar{m}, \Gamma)$, where $\bar{m}$ is the mean and $\Gamma$ the concentration matrix. The probability of observing a transformation given the prior distribution of $m^{k}$ is,

$$
P\left(m^{k} \mid \theta\right)=\frac{\operatorname{det}(\Gamma)^{1 / 2}}{(2 \pi)^{d / 2}} \exp \left(-\frac{1}{2}\left(m^{k}-\bar{m}\right)^{T} \Gamma\left(m^{k}-\bar{m}\right)\right),
$$

where $d=12 N$. Assume that we know $\theta=(\bar{m}, \Gamma)$ and that we want to register with this prior, for this we optimize the MAP estimate,

$$
P\left(m^{k} \mid v^{k}, \theta\right)=\frac{P\left(v^{k} \mid m^{k}, \theta\right) P\left(m^{k} \mid \theta\right)}{P\left(v^{k} \mid \theta\right)},
$$

the optimal solution of $\mathcal{L}\left(m^{k} \mid \theta\right)$ (modulo the constant $\left.P\left(v^{k} \mid \theta\right)\right)$ is,

$$
\check{m}^{k}=(\Sigma+\Gamma)^{-1}\left(\Sigma \hat{m}^{k}+\Gamma \bar{m}\right) .
$$

For our experiments on mandible CT images, we estimate $\Gamma$ using a blockwise pseudoinverse described in the next paragraph. 
Blockwise Pseudoinverse of Sample Covariance Matrix for Prior. To estimate $\Gamma$ we take advantage of the region structure, which reflects as a blockwise structure in the covariance matrix, $\operatorname{Cov}=\frac{1}{K} \sum_{k}^{K}\left(\hat{m}^{k}-\bar{m}\right)\left(\hat{m}^{k}-\bar{m}\right)^{T}$, where $\bar{m}$ is the sample mean. We develop a blockwise pseudoinverse, $\Gamma=\operatorname{diag}\left(\lambda_{i}^{+} \otimes C^{-1}\right)$, where ${ }^{+}$is the pseudoinverse, $\lambda_{i}$ is the variance of block $i$ (i.e. region $i$ ), and $C$ is a $12 \times 12$ matrix, representing the metric of the log affine parameters. We optimize, $\left\{\hat{C}, \hat{\lambda}_{i}\right\}=\operatorname{argmin}_{C, \lambda_{i}}=\sum_{i}^{N}\left\|\operatorname{Cov}_{i i}-\lambda_{i} C\right\|^{2}$, in two sequentially steps. In step one, for $C$ with fixed $\forall i: \lambda_{i}=1$, and in step two for $\lambda_{i}$ with fixed $C$ obtained from the previous step:

$$
\text { Step one: } \hat{C}=\frac{\sum_{i=1}^{N} \lambda_{i} \operatorname{Cov}_{i i}}{\sum_{i=1}^{N} \lambda_{i}^{2}}, \quad \text { Step two: } \hat{\lambda}_{i}=\frac{\operatorname{Trace}\left(\operatorname{Cov}_{i i} C\right)}{\operatorname{Trace}\left(C^{2}\right)}
$$

\section{Experiments on Mandible CT Image Data}

In this section, we register 42 mandible CT images to a randomly chosen template and evaluate the resulting transformations. The noise parameter is set to $\sigma_{v}=1.85 \mathrm{~mm}$, and the number of affine components is $N=31$ across 5 levels. To evaluate the influence of the prior, we run five different experiments: no prior, $\lambda_{i}$ scaled by 1,0.1,0.01 and 0.001 . For the evaluation of the robustness we decompose the log affine transformations into three parts: rotation, expansion and translation. The decomposition of the affine part into skew symmetric
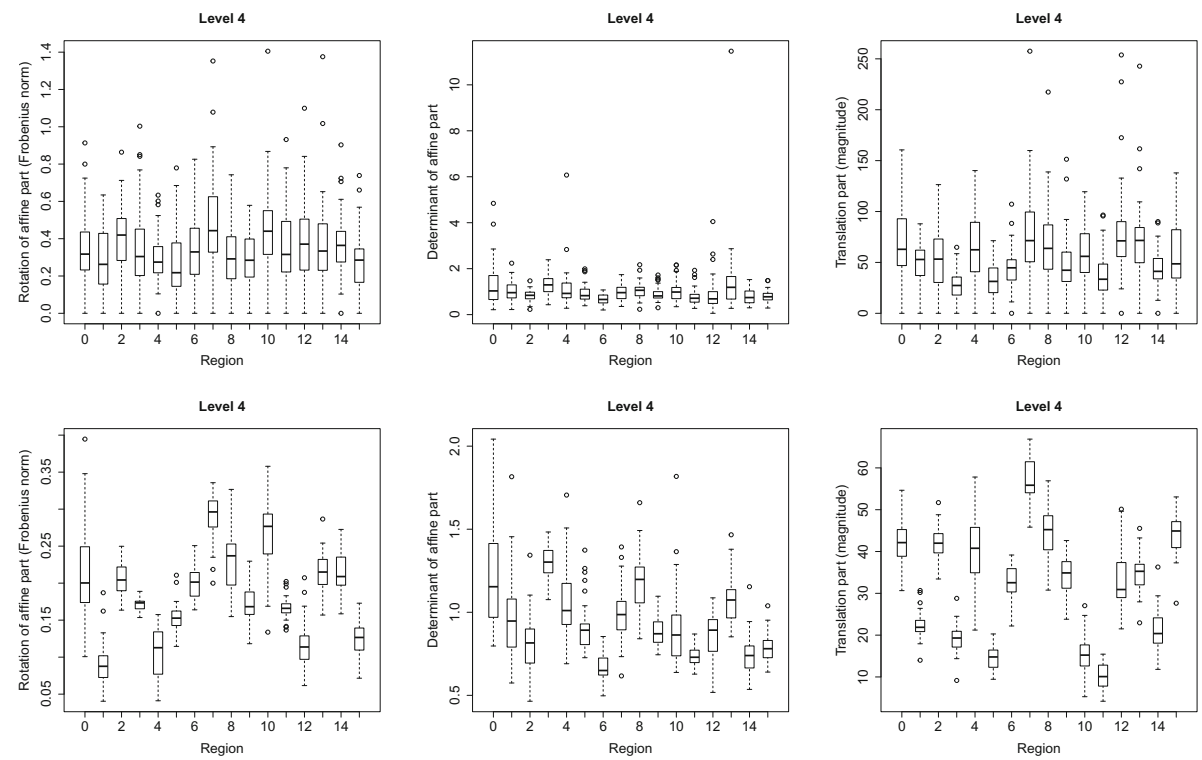

Fig. 2. In top row statistics on transformations obtained with registration without prior and bottom row with prior for level 4 (i.e. 16 regions) 
and symmetric matrices (representing rotation and expansion, respectively) is, $M_{i}=\frac{1}{2}\left(M_{i}-M_{i}^{T}\right)+\frac{1}{2}\left(M_{i}+M_{i}^{T}\right)$, and the Jordan or Schur decomposition to compute the determinant of an affine part, is given by, $\operatorname{det}\left(A_{i}\right)=\exp \left(\operatorname{Trace}\left(M_{i}\right)\right)$.
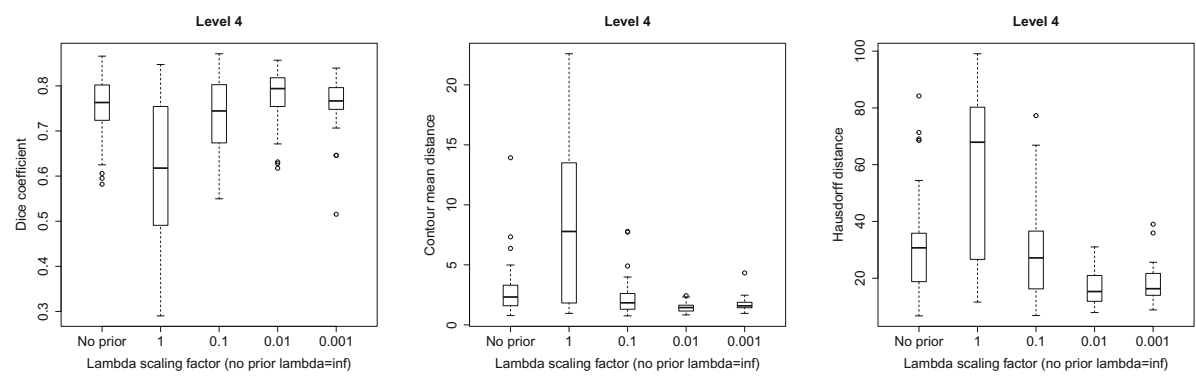

Fig. 3. Comparison of registration accuracy in terms of Dice coefficient, contour mean distance and Hausdorff distance (both in $\mathrm{mm}$ ) at level 4 . The parameter $\lambda_{i}$ was scaled with five different factors to evaluate the influence of the prior.

The robustness results are shown for level 4 (i.e. 16 regions) in Fig. 2, The registrations without prior show outliers and very high expansion of regions up to a determinant of 10 . In the registration with prior (the prior was scaled by 0.01 ), we observe robust expansion and shrinkage factors between 0.5 and 2 . The accuracy of the registration is measured in terms of Dice coefficient of the mask images (semi-manual segmented mask are available for all 42 images), mean contour distance, and Hausdorff distance on the mask contour. In Fig. 3, by scaling the prior with 0.01 all accuracy measure show a favorable median value compared to the without prior registration.

\section{Conclusion}

In this work, we presented a multiscale polyaffine registration method that simultaneously registers all scales. To avoid local solutions we incorporated deformations statistics. The results showed that the registration with prior improves the accuracy while reducing the variability of estimated transformation parameters.

We believe that our approach is more than just an extension of [2]. By considering not only one affine component, but a mixture of components acting at different scales, we are moving the discussion into structured learning, which to our knowledge is a novelty in the medical registration community. In future work, we plan to introduce a sparse representation of anatomical substructures and their connection at different scales, which might uncover structures equivalent to rigid articulated bodies.

Acknowledgements. This work was funded by the Swiss National Science Foundation. 


\section{References}

1. Arsigny, V., Commowick, O., Ayache, N., Pennec, X.: A Fast and Log-Euclidean Polyaffine Framework for Locally Linear Registration. J. Math. Imaging Vis. 33(2), 222-238 (2009)

2. Ashburner, J., Neelin, P., Collins, D.L., Evans, A., Friston, K.: Incorporating Prior Knowledge Into Image Registration. NeuroImage 6(4), 344-352 (1997)

3. Bou-Sleiman, H., Ritacco, L.E., Nolte, L.-P., Reyes, M.: Minimization of IntraOperative Shaping of Orthopaedic Fixation Plates: A Population-Based Design. In: Fichtinger, G., Martel, A., Peters, T. (eds.) MICCAI 2011, Part II. LNCS, vol. 6892, pp. 409-416. Springer, Heidelberg (2011)

4. Buerger, C., Schaeffter, T., King, A.P.: Hierarchical Adaptive Local Affine Registration for Fast and Robust Respiratory Motion Estimation. Med. Image Anal. 15(4), 551-564 (2011)

5. Commowick, O., Arsigny, V., Isambert, A., Costa, J., Dhermain, F., Bidault, F., Bondiau, P.Y., Ayache, N., Malandain, G.: An Efficient Locally Affine Framework for the Smooth Registration of Anatomical Structures. Med. Image Anal. 12(4), 427-441 (2008)

6. Ellis, E., Moos, K.F., El-Attar, A.: Ten Years of Mandibular Fractures: An Analysis of 2,137 Cases. Oral Surg. Oral Med. Oral Pathol. 59(2), 120-129 (1985)

7. Lovald, S., Baack, B., Gaball, C., Olson, G., Hoard, A.: Biomechanical Optimization of Bone Plates Used in Rigid Fixation of Mandibular Symphysis Fractures. Journal of Oral and Maxillofacial Surgery 68(8), 1833-1841 (2010)

8. Marsland, S., Twining, C.J., Taylor, C.J.: A Minimum Description Length Objective Function for Groupwise Non-Rigid Image Registration. Image Vision Comput 26(3), 333-346 (2008)

9. Pennec, X., Stefanescu, R., Arsigny, V., Fillard, P., Ayache, N.: Riemannian Elasticity: A Statistical Regularization Framework for Non-linear Registration. In: Duncan, J.S., Gerig, G. (eds.) MICCAI 2005. LNCS, vol. 3750, pp. 943-950. Springer, Heidelberg (2005)

10. Seiler, C., Pennec, X., Reyes, M.: Capturing the Multiscale Anatomical Shape Variability with Polyaffine Transformation Trees. Med. Image Anal. (2012), http://dx.doi.org/10.1016/j.media.2012.05.011

11. Taquet, M., Macq, B., Warfield, S.K.: Spatially Adaptive Log-Euclidean Polyaffine Registration Based on Sparse Matches. In: Fichtinger, G., Martel, A., Peters, T. (eds.) MICCAI 2011, Part II. LNCS, vol. 6892, pp. 590-597. Springer, Heidelberg (2011)

12. Urken, M.L., Weinberg, H., Vickery, C., Buchbinder, D., Lawson, W., Biller, H.F.: Oromandibular Reconstruction Using Microvascular Composite Free Flaps. Report of 71 Cases and a New Classification Scheme for Bony, Soft-Tissue, and Beurologic Defects. Arch. Otolaryngol Head Neck Surg. 117(7), 733-744 (1991)

13. Vercauteren, T., Pennec, X., Perchant, A., Ayache, N.: Diffeomorphic Demons: Efficient Non-Parametric Image Registration. NeuroImage 45(1, suppl. 1), S61S72 (2009) 